\title{
The Upper Envelope of Piecewise Linear Functions: Tight Bounds on the Number of Faces*
}

\author{
Herbert Edelsbrunner \\ Department of Computer Science, University of llinois at Urbana-Champaign, \\ Urbana, IL 61801, USA
}

\begin{abstract}
This note proves that the maximum number of faces (of any dimension) of the upper envelope of a set of $n$ possibly intersecting $d$-simplices in $d+1$ dimensions is $\Theta\left(n^{d} \alpha(n)\right)$. This is an extension of a result of Pach and Sharir [PS] who prove the same bound for the number of $d$-dimensional faces of the upper envelope.
\end{abstract}

\section{Introduction}

This note considers the combinatorial complexity ${ }^{1}$ of the upper envelope of a finite set of (possibly intersecting) $d$-dimensional simplices ${ }^{2}$ in $(d+1)$ dimensional Euclidean space. In order to define the notion of an envelope we think of each $d$-simplex as the graph of a real-valued, linear $d$-variate function. This function, $f$, is defined so that $x_{d+1}=f\left(x_{1}, x_{2}, \ldots, x_{d}\right)$ whenever $\left(x_{1}, x_{2}, \ldots, x_{d}, x_{d+1}\right)$ is in the simplex. If no such $x_{d+1}$ exists we conveniently set $f\left(x_{1}, x_{2}, \ldots, x_{d}\right)=-\infty$. The (upper) envelope of the set of simplices is now the pointwise maximum of all corresponding $d$-variate functions. The (upper) envelope of more general piecewise linear $d$-variate functions is implicitly defined since the graph of every such function is a collection of $d$-dimensional polyhedra

* This work was supported by Amoco Fnd. Fac. Dev. Comput. Sci. 1-6-44862 and by the National Science Foundation under Grant CCR-8714565. Research on the presented result was partially carried out while the author worked for the IBM T. J. Watson Research Center at Yorktown Height, New York, USA.

${ }^{1}$ By the combinatorial complexity we mean the number of faces of any dimension $k<d$. In our analysis we assume that $d$, the number of dimensions, is a fixed constant.

${ }^{2}$ A $d$-dimensional simplex (or $d$-simplex) in $d+1$ dimensions is the intersection of a hyperplane with $d+1$ half-spaces, where a half-space is defined as the set of points on and to one side of a hyperplane. 
which can be decomposed into $d$-simplices. To prove an upper bound on the combinatorial complexity of the envelope of $n d$-simplices we assume without loss of generality that the $d$-simplices are in general position. Among other things this means that the hyperplanes that contain the $d$-simplices are nonvertical. ${ }^{3}$ Other implications of the general position assumption are implicitly used whenever it is convenient.

Let $S$ be such a set of $n d$-simplices in $d+1$ dimensions and let $M_{S}$ be its envelope. If we project every face of $M_{S}$ vertically onto the hyperplane $x_{d+1}=0$ we get a cell complex, ${ }^{4} M_{S}^{*}$, and we denote the number of $k$-faces ${ }^{5}$ of $M_{S}^{*}$ by $\psi_{k}(S)$ for $0 \leq k \leq d$. Formally, we consider the sum of the $\psi_{k}(S)$ as the combinatorial complexity of $M_{S}$. This note proves tight upper bounds for $\psi_{k}^{(d+1)}(n)$, where

$$
\psi_{k}^{(d+1)}(n)=\max \left\{\psi_{k}(S) \mid S \text { a set of } n d \text {-simplices in } d+1 \text { dimensions }\right\}
$$

for all $0 \leq k \leq d$ and constant values of $d$. Prior to this note, tight bounds were known for all $k$ only if $d+1=2,3$ and for $k=d$ if $d>3$. In two dimensions $(d+1=2), S$ is a set of (possibly intersecting) line segments in the plane. Using so-called Davenport-Schinzel sequences of order 3 [HS] and [WS] prove that $\psi_{k}^{(2)}(n)=\Theta(n \alpha(n))$, for $k=0,1$, where $\alpha(n)$ is the extremely slowly growing inverse of Ackermann's function. [PS] proves $\psi_{d}^{(d+1)}(n)=O\left(n^{d} \alpha(n)\right)$ using a divide-and-conquer argument and shows that this upper bound is tight by extending the two-dimensional lower bound construction of [WS] to three and higher dimensions. In $d+1=3$ dimensions the Euler characteristic can be used to extend the upper bound for 2 -faces to 0 -faces (vertices) and 1-faces (edges). In this note we prove the following result.

Theorem. $\psi_{k}^{(d+1)}(n)=\Theta\left(n^{d} \alpha(n)\right)$ for $0 \leq k \leq d$.

In other words, the combinatorial complexity of the envelope of $n d$-simplices in $d+1$ dimensions is proportional to $n^{d} \alpha(n)$ in the worst case. It is easy to verify the lower bound of the theorem. [PS] shows that there is a collection of $n d$-simplices in $d+1$ dimensions such that the number of $d$-faces of the envelope is $\Omega\left(n^{d} \alpha(n)\right)$. The lower bound for $0 \leq k<d$ follows since every $d$-face has at least one $k$-face in its boundary and every $k$-face belongs to the boundary of at most some constant number of $d$-faces, if we assume general position of the $d$-simplices. The constant is linear in $d$. The proof of the upper bound is presented in Section 2 of this note. It is an extension of the divide-and-conquer proof of

\footnotetext{
${ }^{3}$ A hyperplane is nonvertical if it intersects the $(d+1)$ st coordinate axis in a unique point.

${ }^{4}$ A cell complex is a collection of closed convex sets (called faces) of various dimensions such that the relative interiors of the faces partition the space and the intersection of any two faces is again a face.

${ }^{5}$ A maximal connected component, $f$, of the intersection of $M_{S}^{*}$ with a $k$-dimensional affine subspace is a $k$-face of $M_{S}^{*}$ if the interior of $f$ relative to the subspace is nonempty and $f$ is not contained in the relative interior of a $(k+1)$-face of $M_{S}^{*}$.
} 
[PS]. Combinatorial extensions and algorithmic applications of the theorem can be found in [EGS].

\section{Proof of the Theorem}

We first review the main steps of the proof and then describe each step in appropriate detail. Most of the arguments are concerned with a refinement, $\bar{M}_{S}$, of the cell complex $M_{S}^{*}$ in $d$ dimensions. $\vec{M}_{S}$ has the nice property that every face is convex. Being a refinement of $M_{S}^{*}$ the number of faces of $\bar{M}_{S}$ is certainly an upper bound on the number of faces of $M_{S}^{*}$. The overall structure of the proof is inductive over the number of dimensions. In a specific dimension, $d+1$, we use a divide-and-conquer argument, that is, we form subsets of $S$, the set of $d$-simplices, consider the envelopes of these subsets and combine them to get the envelope of $S$. More precisely, we consider the cell complexes $\bar{M}$ of the subsets and combine those to get $\bar{M}_{S}$. The combination makes use of the convexity of $\bar{M}_{s}$ 's faces and the inductively available upper bounds on the combinatorial complexity of envelopes in $d$ dimensions. A careful choice of the subsets of $S$ allows us to prove the upper bound of the theorem for $2 \leq k \leq d$. Finally, we use the Euler characteristic for cell complexes to extend the upper bound to $k=0,1$. The order in which we present the various steps of the proof is different from the order used in this outline.

Definition of $\bar{M}_{S}$. As mentioned above, $\bar{M}_{S}$ is a refinement of $M_{S}^{*}$ which is a cell complex in $d$ dimensions. (The $d$-dimensional space is identified with the hyperplane $x_{d+1}=0$ in $d+1$ dimensions.) Recall that $M_{S}^{*}$ is obtained by projecting every face of $M_{S}$ vertically onto $x_{d+1}=0$. To obtain $\bar{M}_{S}$ from $M_{S}^{*}$ we also project each $d$-simplex in $S$ vertically onto $x_{d+1}=0$ and, in addition, extend each $(d-1)$-face of each projected $d$-simplex to the full hyperplane in $x_{d+1}=0$ that contains it. Thus, $\bar{M}_{S}$ is $M_{S}^{*}$ after superimposing an arrangement ${ }^{6}$ of $(d+1) n$ hyperplanes; the arrangement is denoted by $A_{S}$.

It is convenient to think of $\bar{M}_{S}$ as a refinement of $A_{S}$ : every cell (i.e., $d$-face) of $A_{S}$ is further decomposed by projections of intersections between $d$-simplices. Consider the vertical slab, $V_{c}$, in $d+1$ dimensions whose points project vertically to points of some cell $c$ of $A_{S}$. Restricted to $V_{c}$, a $d$-simplex in $S$ cannot be distinguished from the ( $d$-dimensional) hyperplane that contains the $d$-simplex. It follows that $M_{S}$, the envelope of $S$, restricted to $V_{c}$ is the boundary of the convex polyhedron that is the intersection of the half-spaces bounded from below by the hyperplanes containing the $d$-simplices cutting through $V_{c}$. This implies that in $\vec{M}_{S}$ every cell of $A_{S}$ is further decomposed into convex faces. Consequently, every face of $\bar{M}_{S}$ is convex. We let $\bar{\psi}_{k}(S)$ denote the number of $k$-faces of $\bar{M}_{S}$.

\footnotetext{
${ }^{6}$ An arrangement in $d$ dimensions is the cell complex obtained by dissecting the space with a finite number of hyperplanes. If $n$ is the number of hyperplanes then the number of faces of the arrangement is $O\left(n^{d}\right)$ (see [Grii] and [E]).
} 


\section{Use of the Euler Characteristic}

The Euler characteristic of a cell complex in $d$ dimensions is a linear relation for the numbers of $k$-faces, $0 \leq k \leq d$. For $\bar{M}_{S}$ it has the simple form

$$
\sum_{k=0}^{d}(-1)^{k} \bar{\psi}_{k}(S)=1+(-1)^{d}
$$

since all faces of $\bar{M}_{S}$ are convex and therefore simply connected (see [Gre]). Assuming $\bar{\psi}_{k}(S)=O\left(n^{d} \alpha(n)\right)$ for $2 \leq k \leq d$ we get

$$
\left|\bar{\psi}_{0}(S)-\bar{\psi}_{1}(S)\right|=O\left(n^{d} \alpha(n)\right)
$$

Thus, the number of vertices and edges of $\bar{M}_{S}$ can be asymptotically more than $n^{d} \alpha(n)$ only if their difference is small, that is, $O\left(n^{d} \alpha(n)\right)$. However, by assumption of general position every vertex of $\vec{M}_{S}$ is incident upon $d+1$ edges if it lies inside a cell of $A_{S}$, and between $d+2$ and $2 d$ if it lies on the boundary of a cell of $A_{S}$. In any case, we have

$$
\bar{\psi}_{1}(S) \geq \frac{d+1}{2} \bar{\psi}_{0}(S)
$$

which implies that both $\bar{\psi}_{0}(S)$ and $\bar{\psi}_{1}(S)$ can be at most proportional to their difference, as long as $d \geq 2$. This proves $\bar{\psi}_{k}^{(d+1)}(n)=O\left(n^{d} \alpha(n)\right)$ for $k=0,1$ if the same upper bound holds for $2 \leq k \leq d$.

\section{An Exercise in Solving Recurrence Relations}

Later we prove that indeed $\bar{\psi}_{k}^{(d+1)}(n)=O\left(n^{d} \alpha(n)\right)$ for $2 \leq k \leq d$. The type of recurrence relation that we have to deal with is of the form

$$
T(n)=\left(\begin{array}{c}
m \\
d+1-k
\end{array}\right) \cdot T\left(\frac{d+1-k}{m} \cdot n\right)+O\left(n^{d} \alpha(n)\right),
$$

where $m>d+1-k$ is an integer constant independent of $n$. The solution to this recurrence relation is $O\left(n^{d} \alpha(n)\right)$ if the homogeneous solution is $O\left(n^{d-\varepsilon}\right)$ for some $\varepsilon>0$. We show that $m$ can always be chosen such that this is true.

The homogeneous solution of the above recurrence relation is $n^{\beta}$, with

$$
\beta=\log _{2}\left(\begin{array}{c}
m \\
d+1-k
\end{array}\right) / \log _{2} \frac{m}{d+1-k} .
$$

The requirement $\beta<d$ can be rewritten as

$$
\left(\begin{array}{c}
m \\
d+1-k
\end{array}\right)<\left(\frac{m}{d+1-k}\right)^{d}
$$


which is equivalent to

$$
\frac{(d+1-k)^{d}}{(d+1-k) !}<\frac{m^{d}}{m \cdot(m-1) \cdots(m-d+k)} .
$$

The ratio on the right side has $d$ factors in the numerator and $d+1-k$ factors in the denominator which implies that

$$
\frac{(d+1-k)^{d}}{(d+1-k) !}<m
$$

is sufficient to guarantee $\beta<d$ as long as $d+1-k<d$ which is equivalent to $k \geq 2$. Thus, the recurrence relation solves to $O\left(n^{d} \alpha(n)\right)$ if $k \geq 2$ and $m$ is chosen appropriately. The above calculation shows that choosing $m$ exponentially in $d$ is sufficient.

\section{Adding Hyperplanes}

The final step of the proof (described later) takes the envelopes of a constant number of subsets of $S$ and obtains the envelope of $S$ by combining those envelopes. Let $S_{1}, S_{2}, \ldots, S_{\mu}$ be the subsets of $S$ and consider the cell complexes $\bar{M}_{S_{i}}$ for $1 \leq i \leq \mu$. When we combine those cell complexes it is important that they are refinements of the same arrangement as $\bar{M}_{S}$, namely of $A_{S}$. To satisfy this need, we superimpose $A_{S}$ on $\bar{M}_{S}$, for every $1 \leq i \leq \mu$, and call the resulting cell complex $\bar{M}_{S_{i}}$. Adding hyperplanes to $\bar{M}_{S_{\mathrm{t}}}$ clearly increases the number of faces. We now show that the effect of adding hyperplanes on the number of faces is surprisingly small.

When we add a hyperplane we create new $k$-faces that lie in the hyperplane and we cut old $k$-faces into pairs of new $k$-faces; in the latter case the hyperplane contains a $(k-1)$-face that splits the old $k$-face. Thus, we can estimate the increase in combinatorial complexity from $\bar{M}_{S_{1}}$ to $\bar{M}_{S}$ by counting the faces in the hyperplanes added to $\bar{M}_{S_{1}}$. The number of hyperplanes added to $\bar{M}_{S_{1}}$ is at most $(d+1) n$ and thus linear in the size of $S .^{7}$

Consider now the decomposition of a hyperplane, $h$, in $\overline{\bar{M}}_{S_{i}}$. In order to bound the number of faces in $h$ we use the following auxiliary claim, which we also establish using induction over the number of dimensions. The claim considers cell complexes that are slightly more general than the cell complexes $\bar{M}$.

Claim. Let $S$ be a finite set of d-simplices in $d+1$ dimensions, let $\bar{M}_{S}$ be the cell complex in $d$ dimensions as defined earlier, and let $\bar{M}$ be $\bar{M}_{S}$ after adding a finite number of hyperplanes (in d dimensions). The number of faces of $\bar{M}$ is $O\left(N^{d} \alpha(N)\right)$, where $N$ is the number of $d$-simplices in $S$ plus the number of hyperplanes added to $\bar{M}_{S}$.

\footnotetext{
${ }^{7}$ Some of the hyperplanes of $A_{S}$ are already present in $\bar{M}_{S_{7}}$ and do not have to be added.
} 
If $d=1, S$ is a finite set of line segments in the plane. The vertical projection of the upper envelope of $S$ is a decomposition of the $x_{1}$-axis into intervals. [WS] establishes that the number of intervals is $O(n \alpha(n))$ if $n=|S|$. If we add $N-n$ points to the subdivision of the $x_{1}$-axis we get at most $O(n \alpha(n)+N)$ intervals which is smaller than $O(N \alpha(N))$ and thus the claim is correct for $d=1$.

We now come back to hyperplane $h$ which intersects the other hyperplanes in a $(d-1)$-dimensional arrangement consisting of $O\left(n^{d-1}\right)$ faces. The decomposition of $h$ in $\bar{M}_{S}$ is a refinement of this arrangement which can be obtained from a cross-section of $M_{S}$ as follows. Let $h^{\prime}$ be the vertical hyperplane in $d+1$ dimensions whose intersection with $x_{d+1}=0$ is $h$. The cross-section $M_{s} \cap h^{\prime}$ is the envelope of $O(n)(d-1)$-simplices ${ }^{8}$ in $h^{\prime}$ which has $O\left(n^{d-1} \alpha(n)\right)$ faces by inductive assumption (the above claim for $(d-1)$-simplices in $d$ dimensions). Inductively, we can also assume that the decomposition of $h$ in $\bar{M}_{S_{\text {, }}}$ (which we obtain by superimposing the vertical projection of the cross-section with the arrangement in $h$ described earlier) has at most $O\left(n^{d-1} \alpha(n)\right)$ faces. Thus, the total number of faces in the cell complexes $\dot{M}$ (taken over all sets $S_{i}$ for $1 \leq i \leq \mu$ ) is at most $O\left(n^{d} \alpha(n)\right)$ larger than the total number of faces of the cell complexes $\bar{M}$ (taken over the same collection of sets).

Notice that the argument makes no use of the fact that every hyperplane added to $\bar{M}_{S_{1}}$ contains a $(d-1)$-face of the vertical projection of a $d$-simplex in $S_{i}$. It can therefore be applied to any odd hyperplane that we like to add. This is important for proving the claim for $d+1$ dimensions which can thus be done along the same lines.

\section{Combining Envelopes}

For this step of the proof it is important that $M_{S}$, the envelope of $S$, restricted to a vertical slab defined by a cell of $A_{S}$, is the lower boundary of a convex polyhedron. Thus, every face is convex and every intersection of $d+1-k d$ simplices (for $0 \leq k \leq d$ ) contains at most one $k$-face within this slab. Let us now fix $k$ to some integer between 2 and $d$ including the limits. We partition $S$ into $m>d+1-k$ subsets of approximately equal sizes ${ }^{9}$ and then form

$$
\mu=\left(\begin{array}{c}
m \\
d+1-k
\end{array}\right)
$$

sets of size approximately $n \cdot(d+1-k) / m$ by merging every combination of $d+1-k$ subsets. For example, if $k=d$ then the new sets are the original $m$ subsets, and if $k=d-1$ the sets are the unions of any two original subsets. It is important to see that any $(d+1-k)$-tuple of $d$-simplices is contained in at least one of the $\mu$ sets.

\footnotetext{
${ }^{8} h^{\prime}$ intersects a $d$-simplex in a $(d-1)$-dimensional convex polytope which can be decomposed into a constant number of $(d-1)$-simplices.

${ }^{9} S$ can be partitioned such that the sizes of any two subsets differ by at most 1 .
} 
We now come back to $M_{S}$, the envelope of $S$, restricted to the vertical slab, $V_{c}$, defined by cell $c$ of the arrangement $A_{S}$ in $x_{d+1}=0$. This restricted part of $M_{S}$ corresponds to the decomposition of $c$ induced by $\bar{M}_{S}$. We consider the $\mu$ sets formed above and denote them by $S_{1}, S_{2}, \ldots, S_{\mu}$. If a $k$-face $f$ of $\bar{M}_{S}$ lies inside $c$, then it is contained in the projection of the intersection of some $d+1-k$ $d$-simplices $s_{1}, s_{2}, \ldots, s_{d+1-k}$. There is at least one index $j, 1 \leq j \leq \mu$, such that $S$, contains all those simplices. By convexity, $\bar{M}_{S}$, restricted to $c$ has a $k$-face $g$ that contains $f ; g$ is also contained in the projection of $s_{1} \cap s_{2} \cap \cdots \cap s_{d+1-k}$. It follows that the number of $k$-faces of $\bar{M}_{S}$ within $c$ is at most the total number of $k$-faces of $\bar{M}_{S_{1}}, \bar{M}_{S_{2}}, \ldots, \bar{M}_{S_{\mu}}$ in $c$. The total number of $k$-faces of $\bar{M}_{S}$ is thus at most the sum of the numbers of $k$-faces of $\overline{\bar{M}}_{S_{1}}$ through $\bar{M}_{S_{\mu}}$. By the argument in the previous step of the proof we therefore get

$$
T(n)=\left(\begin{array}{c}
m \\
d+1-k
\end{array}\right) T\left(\frac{d+1-k}{m} \cdot n\right)+O\left(n^{d} \alpha(n)\right),
$$

where $T(n)$ is the maximum number of $k$-faces of $\vec{M}_{S}$, that is, $T(n)=\bar{\psi}_{k}^{(d+1)}(n)$. The analysis of this recurrence relation presented earlier implies that the constant $m$ can be chosen so that the solution is $O\left(n^{d} \alpha(n)\right)$. This implies

$$
\bar{\psi}_{k}^{(d+1)}(n)=O\left(n^{d} \alpha(n)\right) \quad \text { for } \quad 2 \leq k \leq d .
$$

The same bound for $k=0,1$ is now implied by our considerations of the Euler characteristic of $\bar{M}_{S}$. This completes the proof of the theorem.

\section{References}

[E] Edelsbrunner, H., Algorithms in Combinatorial Geometry, Springer-Verlag, Heidelberg, 1987.

[EGS] Edelsbrunner, H., Guibas, L. J., and Sharir, M., The upper envelope of piecewise linear functions: algorithms and applications, Discrete Comput. Geom., to appear.

[Gre] Greenberg, M. J., Lectures on Algebraic Topology, Benjamin, Reading, MA, 1967.

[Grü] Grünbaum, B., Convex Polytopes, Wiley, Chichester, 1967.

[HS] Hart, S. and Sharir, M., Nonlinearity of Davenport-Schinzel sequences and of generalized path compression schemes, Combinatorica 6 (1986), 151-177.

[PS] Pach, J. and Sharir, M., The upper envelope of piecewise linear functions and the boundary of a region enclosed by convex plates: combinatorial analysis, Discrete Comput. Geom., to appear.

[WS] Wiernik, A. and Sharir, M., Planar realization of nonlinear Davenport-Schinzel sequences by segments, Discrete Comput. Geom. 3 (1988), 15-47.

Received January 11, 1988. 